\title{
Plasmonic Integrated Multimode Filter
}

\author{
Nicolás Abadía ${ }^{1,2, *}$, Alireza Samani ${ }^{3}$, Emmanuel D. Le Boulbar ${ }^{1,2}$, David Hayes ${ }^{1,2}$, and Peter M. Smowton ${ }^{1,2}$ \\ ${ }^{1}$ School of Physics and Astronomy, Cardiff University, The Parade, Cardiff CF24 3AA, United Kingdom \\ ${ }^{2}$ Institute for Compound Semiconductors, Cardiff University, The Parade, Cardiff CF24 3AA, United Kingdom \\ ${ }^{3}$ Quantum Matter Institute, University of British Columbia, Vancouver, British Columbia V6T 1Z4, Canada \\ Tel: +44 (0)29 2087 4458, e-mail: AbadiaN@cardiff.ac.uk
}

\begin{abstract}
A CMOS-compatible plasmonic multimode filter capable of working in the O, E, S, C, L, and U optical communication bands is analysed. The device consists of a hybrid plasmonic waveguide with metal segments. By properly designing the segments, it is possible to pass a particular mode and diffract the others. The results of the analysis show that the device has an extinction ratio over $20 \mathrm{~dB}$ and an insertion loss of less than $1.6 \mathrm{~dB}$ across the mentioned optical communication bands.
\end{abstract}

Keywords: Integrated optics, polarization-selective devices, plasmonics, silicon photonics.

\section{INTRODUCTION}

In complex photonic integrated circuits, it is important to control the polarization and to filter modes when using multimode waveguides and devices. Polarization control is critical in polarization diversity circuits in order to handle the polarization dependent loss, the polarization mode dispersion [1] and to split different polarizations within the circuit [2]. Those functions are normally performed with polarization rotators and polarization beam splitters [3],[4].

In other applications, it is desirable that the photonic integrated circuit works using one mode: mainly the fundamental TE mode. In such a case, devices like the TE-pass polarizer [5],[6] are exploited. In a similar way, TM-pass polarizers [7],[8] are also employed when using the fundamental TM mode.

Another possible application is to filter the fundamental TE mode from the other modes when routing the photonic integrated circuit with multimode waveguides or when injecting light by the use of edge-coupling techniques. In this case, the fundamental TE or TM mode is filtered from the rest of the modes. The advantages of silicon photonics employing multimode devices are driving interest and ongoing research [9]. In this work, we propose a multimode filter that can pass any particular mode while filtering the others. It can be used for polarization handling and in multimode circuits.

To the best of our knowledge, there are not specific performance requirements for multimode filters, so, we aim to achieve similar values to the integrated TM- and TE-pass polarizers. Regarding the characteristics of the TM- and TE-pass polarizers, it is desired that they have a high polarization extinction ration (PER), a low insertion loss (IL), a compact footprint and a broad bandwidth. Typical values are $P E R=20 \mathrm{~dB}, I L=2-3 \mathrm{~dB}$ and bandwidths around 50-100 $\mathrm{nm}$ [8]. A large bandwidth is desired in order to use the device in the coherent receiver, WDM, and FDM. Nevertheless, it will be useful to have a device that works across all communication bands (O, E, S, C, L, and U). The O, E, C, and L bands [10],[11] are commonly used in germanium and silicon photonics. Other applications including wavelength converters use $\mathrm{S}$ and $\mathrm{U}$ bands [12]. A further consideration is that the fabrication of the multimode filter should be Complementary metal-oxide-semiconductor (CMOS) processes, firstly to allow integration with other silicon photonic devices, and secondly to exploit existing manufacturing capability for future mass production.

Consequently, we will target a multimode filter with similar performance that of TE-pass polarizers with an extinction ratio of $20 \mathrm{~dB}$, insertion loss $<1 \mathrm{~dB}$, working in several optical bands (O, E, S, C, L, and U), CMOS compatibility, and having a compact footprint.

In this work, we numerically design a CMOS compatible plasmonic multimode filter based on a hybrid plasmonic waveguide (HPW) with the metal segmented to act as a grating. We design the grating to pass the desired mode and diffract the other modes supported in the multimode waveguide. The proposed solution can work over the O, E, C, L, S, and U optical communication bands with an extinction ratio larger than $20 \mathrm{~dB}$ and an insertion loss of less than $1.6 \mathrm{~dB}$. The structure has a length smaller than $6 \mu \mathrm{m}$ and it can be excited from either a silicon photonic dielectric waveguide or a hybrid plasmonic waveguide. Hence, the same structure can be exploited in either a photonic or a plasmonic integrated circuit [6].

\section{OPERATIONAL PRINCIPLE}

The structure of the proposed device is presented in Fig. 2, consisting of an HPW with the metal segmented. In Fig. 2(a), the structure of the photonic multimode filter is presented with an input/output dielectric Si waveguide. The same device concept can be used in a plasmonic circuit, in Fig. 2(b) the device has an input/output HPW. The operational principle relies on the design of the metallic segments that act as a grating. The grating can be designed to pass a particular mode e.g. the fundamental $\mathrm{TE}_{0}$ mode and diffract the other modes in a multimode 
waveguide. To design and optimize the structure, we employed a genetic search algorithm [13]. A manual method similar to our TE-pass polarizer [6], can also be used. In this case, the work is extended to any particular mode. The design and optimization process involves the calculation of $T_{\text {initial }}, T_{\text {metal }} . T_{\text {dielectric }}, h_{\text {gap, }}$ and the number of metallic segments represented in Fig. 2(a)

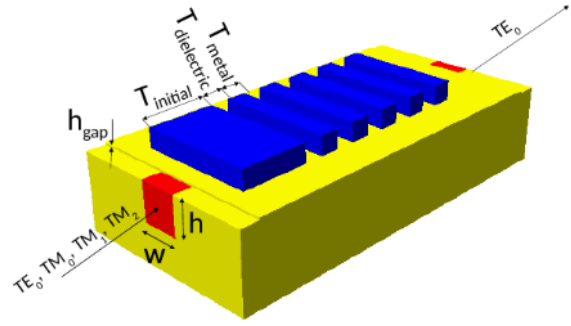

(a)

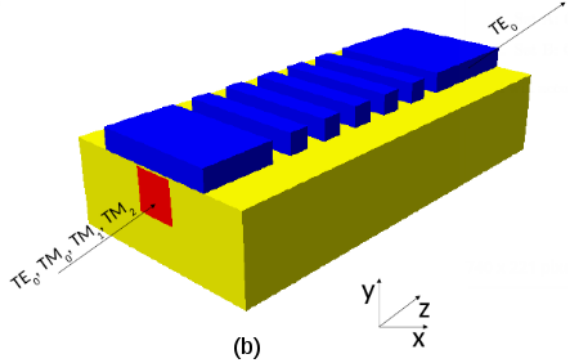

(b)

Figure 1. Proposed plasmonic multi-mode filter: (a) excited from a dielectric silicon photonic waveguide and (b) excited from an HPW.

In order to be CMOS compatible we chose the following materials: $\mathrm{Si}, \mathrm{SiO}_{2}$, and copper $(\mathrm{Cu})$. We selected $\mathrm{Cu}$ over aluminium due to the lower plasmonic propagation losses in $\mathrm{Cu}$ [11].

\section{PERFORMANCE}

We will show the performance of the multimode filter when used in a photonics circuit with multimode photonic waveguides. As an example, we selected a Si waveguide with $w=470 \mathrm{~nm}$ and $h=400 \mathrm{~nm}$. This waveguide supports the following modes: $\mathrm{TE}_{0}, \mathrm{TM}_{0}, \mathrm{TM}_{1}$, and $\mathrm{TE}_{1}$. Other multimode waveguides can also be used.

The dimensions of the multimode filter were optimized to select the $\mathrm{TE}_{0}$ mode and diffract the $\mathrm{TM}_{0}, \mathrm{TM}_{1}$ and $\mathrm{TE}_{1}$ modes. The results of the optimization process gives $T_{\text {initial }}=2 \mu \mathrm{m}, T_{\text {metal }}=0.5 \mu \mathrm{m}, T_{\text {dielectric }}=0.51 \mu \mathrm{m}, h_{\text {gap }}=$ $15 \mathrm{~nm}$, and 7 metallic segments, which gives a total device length of $6.5 \mu \mathrm{m}$. The propagating electric fields of the different modes are plotted in Fig. 2(a)-(d). It is possible to observe how the $\mathrm{TE}_{0}$ mode propagates in the $\mathrm{z}$ direction while the $\mathrm{TM}_{0}, \mathrm{TM}_{1}$, and $\mathrm{TE}_{1}$ diffract. The different polarization extinction ratios with respect to $\mathrm{TE}_{0}$ are represented in Fig. 2(e).
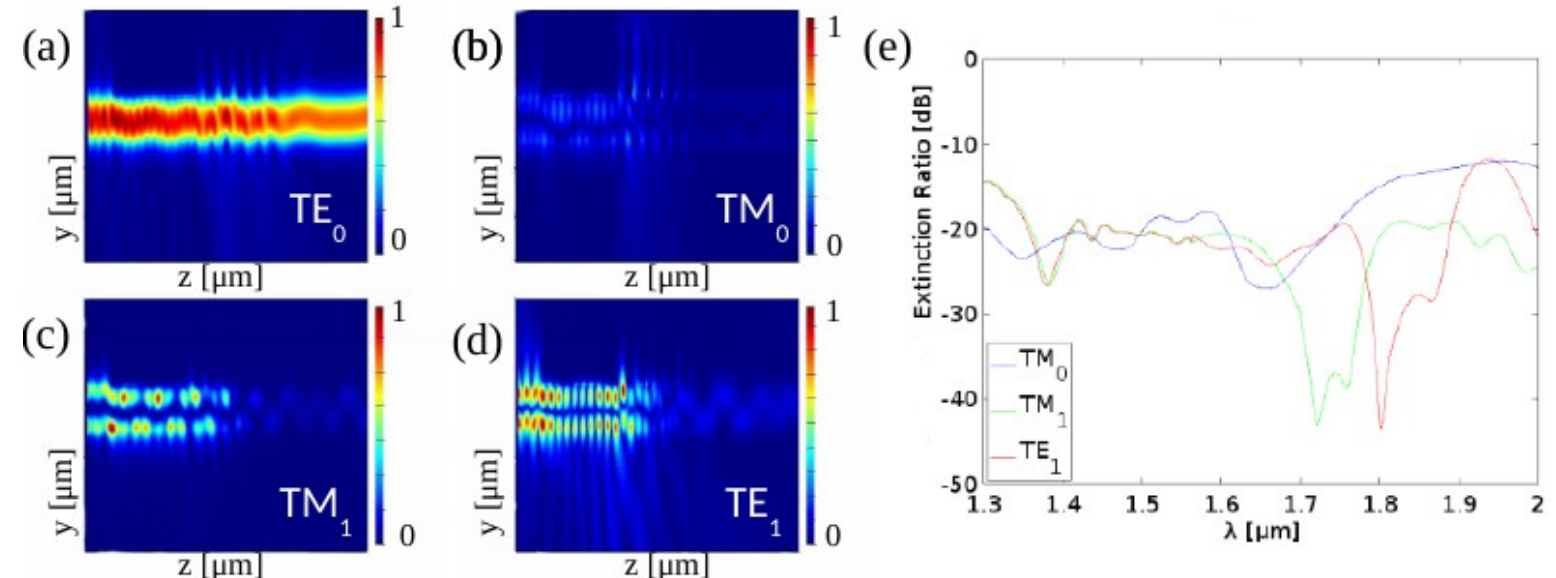

Figure 2: (a) Electric field of the propagation of the TE $E_{0}$ mode, (b) electric field of the propagation of the diffracted $T M_{0}$ mode, (c) $T M_{1}$ mode and (d) $T E_{1}$ mode. (e) Polarization extinction ratios of $T M_{0}, T M_{1}$, and $T E_{1}$ modes versus wavelength.

From Fig. 2(e), it is possible to observe that the proposed device can work in different optical communication bands with a polarization extinction ratio over $20 \mathrm{~dB}$ and insertion loss below 1.6 dB. The insertion loss and the polarization extinction ratios across the different optical communication bands are summarized in Table 1.

Table 1: Estimated insertion loss and polarization extinction ratios across several optical bands. 


\begin{tabular}{|c|c|c|c|c|c|c|}
\hline Band & $\mathrm{O}$ & $\mathrm{E}$ & S & $\mathrm{C}$ & $\mathrm{L}$ & $\mathrm{U}$ \\
\hline $\mathrm{IL}$ & $0.7 \mathrm{~dB}$ & $1 \mathrm{~dB}$ & $1.3 \mathrm{~dB}$ & $1.3 \mathrm{~dB}$ & $1.5 \mathrm{~dB}$ & $1.6 \mathrm{~dB}$ \\
\hline $\operatorname{PER}\left(\mathrm{TM}_{0} / \mathrm{TE}_{0}\right)$ & $20 \mathrm{~dB}$ & $21 \mathrm{~dB}$ & $21 \mathrm{~dB}$ & $19 \mathrm{~dB}$ & $19 \mathrm{~dB}$ & $27 \mathrm{~dB}$ \\
\hline $\operatorname{PER}\left(\mathrm{TM}_{1} / \mathrm{TE}_{0}\right)$ & $20 \mathrm{~dB}$ & $21 \mathrm{~dB}$ & $44 \mathrm{~dB}$ & $46 \mathrm{~dB}$ & $22 \mathrm{~dB}$ & $24 \mathrm{~dB}$ \\
\hline $\operatorname{PER}\left(\mathrm{TM}_{2} / \mathrm{TE}_{0}\right)$ & $21 \mathrm{~dB}$ & $22 \mathrm{~dB}$ & $26 \mathrm{~dB}$ & $25 \mathrm{~dB}$ & $35 \mathrm{~dB}$ & $40 \mathrm{~dB}$ \\
\hline Wavelength & $1.31 \mu \mathrm{m}$ & $1.4 \mu \mathrm{m}$ & $1.5 \mu \mathrm{m}$ & $1.55 \mu \mathrm{m}$ & $1.6 \mu \mathrm{m}$ & $1.65 \mu \mathrm{m}$ \\
\hline
\end{tabular}

This concept can be extended to pass any particular mode in a multimode waveguide and filter the others by the proper design of the metallic segments of the HPW [8]. The same design can be used either in a photonic or a plasmonic circuit. This multimode filter has several applications when used in multimodal circuits [9].

\section{CONCLUSIONS}

In this work, the methodology to design a CMOS compatible multimode filter consisting of a hybrid plasmonic waveguide with the segmented metal is presented. By carefully designing the segments, it will be possible to pass a particular mode and filter out others unwanted modes. The example considered here is capable of working in the $\mathrm{O}, \mathrm{E}, \mathrm{S}, \mathrm{C}, \mathrm{L}$, and $\mathrm{U}$ optical communication bands with an extinction ratio over $20 \mathrm{~dB}$ and an insertion loss smaller than $1.6 \mathrm{~dB}$ when filtering out the $\mathrm{TE}_{1}, \mathrm{TM}_{0}$, and $\mathrm{TM}_{1}$ modes.

\section{ACKNOWLEDGEMENTS}

We are grateful for support from the Future Compound Semiconductor Manufacturing Hub (CS Hub) funded by EPSRC grant reference EP/P006973/1.

\section{REFERENCES}

[1] K. Takiguchi, K. Okamoto, S. Suzuki, and Y. Ohmori: Planar lightwave circuit optical dispersion equalizer, in IEEE Photonics Technology Letters, , 86-88, 1994.

[2] Y. Huang, S. Zhu, H. Zhang, T.-Y. Liow, and G.-Q. Lo: CMOS compatible horizontal nanoplasmonic slot waveguides TE-pass polarizer on silicon-on-insulator platform, in Optics Express, 21(10) 12790-12796, 2013.

[3] N. Abadía, X. Dai, Q. Lu, W.-H. Guo, D. Patel, D. V. Plant, and J. F. Donegan: Highly fabrication tolerant InP based polarization beam splitter based on p-i-n structure, in Optics Express, 25(9) 10070-10077, 2017.

[4] N. Abadia, Xiangyang Dai, Q. Lu, W.-H. Guo, E. El-Fiky, D. V. Plant, and J. F. Donegan: Novel polarization beam splitter based on p-i-n structure for an indium phosphide platform, in Proc. ICTON, Girona, Spain, Jun. 2017, paper Tu.A5.1.

[5] Y. Xiong, D. X. Xu, J. H. Schmid, P. Cheben, and W. N. Ye: High extinction ratio and broadband silicon TE-pass polarizer using subwavelength grating index engineering, in IEEE Photonics Journal, 7(5) 7802107, 2015.

[6] Nicolás Abadía, Md. Ghulam Saber, Frank Bello, Alireza Samani, Eslam El-Fiky, Yun Wang, John F. Donegan, and David V. Plant: CMOS-compatible multi-band plasmonic TE-pass polarizer, in Optics Express, 26 30292-30304, 2018.

[7] X. Guan, P. Chen, S. Chen, P. Xu, Y. Shi, and D. Dai: Low-loss ultracompact transverse-magnetic-pass polarizer with a silicon subwavelength grating waveguide, in Optics Letters, 39(15) 4514-4517, 2014.

[8] M. G. Saber, N. Abadía, and D. V. Plant: CMOS compatible all-silicon TM pass polarizer based on highly doped silicon waveguide, in Optics Express, 26(16) 20878-20887, 2018.

[9] C. Li, D. Liu, D. Dai: Multimode silicon photonics, in Nanophotonics, 8(2) pp. 227-247, 2018

[10] N. Abadía, T. Bernadin, P. Chaisakul, S. Olivier, D. Marris-Morini, R. Espiau de Lamaëstre, J. C. Weeber, and L. Vivien: Low-Power consumption Franz-Keldysh effect plasmonic modulator, in Optics Express, 22(9) 11236-11243, 2014.

[11] N. Abadía, S. Olivier, D. Marris-Morini, L. Vivien, T. Bernadin, and J. C. Weeber: A CMOS-compatible Franz-Keldysh effect plasmonic modulator,” in Proc. GFP, Paris, France, Aug. 2014, pp. 63-64

[12] M. A. Foster, A. C. Turner, R. Salem, M. Lipson, and A. L. Gaeta: Broad-band continuous-wave parametric wavelength conversion in silicon nanowaveguides, in Optics Express, 15(20) 12949-12958, 2007.

[13] G. Jain, M. J. Wallace, Q. Lu, W. Guo, and J. F. Donegan: Design Optimization for Semiconductor Lasers with High-Order Surface Gratings having Multiple Periods, in Conference on Lasers and Electro-Optics, OSA Technical Digest (online) (Optical Society of America, 2018), paper JTu2A.26. 